Quim. Nova, Vol. 31, No. 5, 1230-1236, 2008

\title{
EXTRAÇÃo LÍQUIDO-LÍQUIDO DE FERRO(III) E TITÂNIO(IV) PELO ÁCIDO BIS-(2-ETIL-HEXIL) FOSFÓ- RICO (D2EHPA) EM MEIO DE ÁCIDO SULFÚRICO
}

\author{
Glauco Corrêa da Silva e José Waldemar Silva Dias da Cunha \\ Departamento de Química e Materiais Nucleares, Instituto de Engenharia Nuclear, CP 68456, 21941-909 Rio de Janeiro - RJ, \\ Brasil \\ Jo Dweck \\ Departamento de Processos Inorgânicos, Escola de Química, Universidade Federal do Rio de Janeiro, Av. Athos da Silveira \\ Ramos, 149, CT, Bloco E, 21941-909 Rio de Janeiro - RJ, Brasil \\ Julio Carlos Afonso* \\ Departamento de Química Analítica, Instituto de Química, Universidade Federal do Rio de Janeiro, Av. Athos da Silveira \\ Ramos, 149, CT, Bloco A, 21941-909 Rio de Janeiro - RJ, Brasil
}

Recebido em 2/4/07; aceito em 20/9/07; publicado na web em 2/4/08

\begin{abstract}
LIQUID-LIQUID EXTRACTION (LLE) OF Fe(III) AND Ti(IV) BY BIS-(2-ETHYL-HEXYL) PHOSPHORIC ACID (D2EHPA) IN SULFURIC ACID MEDIUM: This work presents a study on the separation of Fe(III) and Ti(IV) from sulfuric acid leaching solutions of ilmenite $\left(\mathrm{FeTiO}_{3}\right)$ using liquid-liquid extraction with D2EHPA in n-dodecane as extracting agent. The distribution coefficients $\left(\mathrm{K}_{\mathrm{D}}\right)$ of the elements related to free acidity and concentration of $\mathrm{Fe}(\mathrm{III})$ and Ti(IV) were determined. Free acidity was changed from $3 \times 10^{-2}$ to $11.88 \mathrm{~mol} \mathrm{~L}^{-1}$ and D2EHPA concentration was fixed at $1.5 \mathrm{~mol} \mathrm{\textrm {L } ^ { 1 }}$. Recovery of final products as well as recycling of wastes generated in the process were also investigated. The LLE process as a feasible alternative to obtain high-purity $\mathrm{TiO}_{2}$.
\end{abstract}

Keywords: titanium; solvent extraction; D2EHPA

\section{INTRODUÇÃO}

O elemento titânio é o $16^{\circ}$ mais abundante $(0,6 \% \mathrm{~m} / \mathrm{m})$ da crosta terrestre. ${ }^{1} \mathrm{O}$ Brasil é detentor das maiores reservas mundiais desse metal na forma de anatásio $\left(\beta-\mathrm{TiO}_{2}\right)$, concentradas nos estados de Minas Gerais e Goiás. O depósito mais importante de ilmenita e rutilo $\left(\alpha-\mathrm{TiO}_{2}\right)$ situa-se em Mataraca, estado da Paraíba. ${ }^{2} \mathrm{~A}$ ilmenita é um titanato de $\mathrm{Fe}(\mathrm{II}), \mathrm{FeTiO}_{3}$, podendo possuir teores variáveis de $\mathrm{Fe}(\mathrm{III})$ e outros elementos, tais como $\mathrm{Al}, \mathrm{Ca}, \mathrm{Cr}, \mathrm{Mg}, \mathrm{Mn}, \mathrm{Nb}$, $\mathrm{P}$, Si e V. As areias monazíticas brasileiras, encontradas principalmente em regiões costeiras, são compostas basicamente de sílica $\left(\mathrm{SiO}_{2}\right)$, contendo minerais acessórios como espinélios $\left(\mathrm{Me}^{\mathrm{II}} \mathrm{Me}_{2}^{\mathrm{III}}\right.$ $\left.\mathrm{O}_{4}\right)$, granadas $\left(\mathrm{Me}_{3}^{\mathrm{II}} \mathrm{Me}_{2}{ }_{2}\left(\mathrm{SiO}_{4}\right)_{3}\right)$, rutilo, zirconita $\left(\mathrm{ZrSiO}_{4}\right)$, monazita $\left(\mathrm{LnPO}_{4}\right)$ e ilmenita. Esta última compõe cerca de $20 \%$ $\mathrm{m} / \mathrm{m}$ da areia, e é beneficiada pelas Indústrias Nucleares Brasileiras (INB), de onde é extraída a monazita, empregada para obtenção dos lantanídios Th e U. A ilmenita é obtida como subproduto, junto com o rutilo e a zirconita, sendo vendida como minério de Ti de baixo valor comercial.

Com o desenvolvimento da tecnologia para emprego do $\mathrm{Ti}$, suas ligas e do $\mathrm{TiO}_{2}$ surgiu a necessidade de estabelecer metodologias de produção e purificação em larga escala desses compostos, de forma a atender à crescente demanda nos mais variados graus de pureza para um mercado emergente e extremamente diversificado. ${ }^{3-9}$ As reservas conhecidas dos óxidos de titânio (rutilo/anatásio) tendem a diminuir. Para a produção do chamado "rutilo sintético", outros minérios de Ti devem ser utilizados, sendo a ilmenita a escolha imediata devido à abundância na natureza. ${ }^{10}$

A ilmenita exige para sua abertura a utilização de processos drásticos, tais como digestão com ácidos concentrados a quente $(\mathrm{HCl}$,

*e-mail: julio@iq.ufrj.br
$\left.\mathrm{H}_{2} \mathrm{SO}_{4}, \mathrm{H}_{2} \mathrm{~F}_{2}\right) ;, 5,6,10-15$ fusão com substâncias de caráter ácido ( $\mathrm{KHSO}_{4}$, $\left.\mathrm{Na}_{2} \mathrm{~S}_{2} \mathrm{O}_{7}\right) ;{ }^{4}$ alcalinas $\left(\mathrm{KOH}, \mathrm{Na}_{2} \mathrm{CO}_{3}, \mathrm{CaO}\right) ;{ }^{7}, 16$ redutoras $\left(\mathrm{C}, \mathrm{S}_{8}\right)^{4,17}$ ou complexantes $\left(\mathrm{CaF}_{2}, \mathrm{NH}_{4} \mathrm{~F}, \mathrm{NH}_{4} \mathrm{HF}_{2}\right){ }^{4} \mathrm{~A}$ principal rota de produção industrial do $\mathrm{TiO}_{2}$ é o chamado processo sulfato (hidrólise térmica), que emprega $\mathrm{H}_{2} \mathrm{SO}_{4}$ concentrado. As etapas principais são: abertura do minério, clarificação e filtração, hidrólise térmica, lavagem e filtração, secagem e calcinação, acabamento e moagem. O controle de temperatura é essencial neste processo porque a digestão da ilmenita é fortemente exotérmica. ${ }^{6,11}$ Um sério problema desse processo é a considerável geração de resíduos. ${ }^{4,8,16}$ A produção de $1 \mathrm{t}$ de $\mathrm{TiO}_{2}$ sintético gera cerca de 3,5 $\mathrm{t}$ de rejeitos.

A separação do Fe do Ti presente no minério é a etapa primordial para se obter $\mathrm{TiO}_{2}$ de alta pureza. Soluções de abertura clorídrica aquecidas à ebulição provocam a hidrólise do Ti(IV) precipitando o $\mathrm{TiO}_{2}{ }^{18-20} \mathrm{~A}$ hidrólise é facilitada pela adição de $\mathrm{H}_{2} \mathrm{SO}_{4} \cdot{ }^{20}$ Este processo não elimina completamente o Fe do precipitado. Os métodos mais eficientes mantêm-no em solução antes da hidrólise do Ti(IV), pela redução do $\mathrm{Fe}(\mathrm{III})$ a $\mathrm{Fe}(\mathrm{II})$ mediante agentes redutores, como $\mathrm{H}_{2} \mathrm{~S},\left(\mathrm{NH}_{4}\right)_{2} \mathrm{~S}, \mathrm{SO}_{2}$ e $\mathrm{Na}_{2} \mathrm{~S}_{2} \mathrm{O}_{3}{ }^{4,8}$ Quando a solução é hidrolisada o $\mathrm{Fe}$ (II) não precipita com o TiO ${ }_{2}{ }^{20,21}$ Ele também pode ser mantido em solução com adição de ácidos complexantes (cítrico, oxálico ou tartárico) antes da hidrólise do Ti(IV)..$^{1,21,22}$

Como alternativa para reduzir a enorme geração de resíduos por massa de $\mathrm{TiO}_{2}$ produzida, as atenções vêm se voltando para a aplicação da técnica de extração do Ti por extratantes dissolvidos em solventes orgânicos (extração líquido-líquido ou ELL), seguindo tendência observada para o processamento de outros minérios e resíduos tecnológicos (baterias, catalisadores etc.). O D2EHPA [ácido bis(2-etil-hexil) fosfórico] é um dos mais importantes extratantes nos estudos de isolamento do Ti do $\mathrm{Fe}$, face à resistência à hidrólise em meio ácido durante a extração do Ti e à extração preferencial 
deste sobre o $\mathrm{Fe}^{23-31} \mathrm{O}$ principal meio utilizado é o $\mathrm{H}_{2} \mathrm{SO}_{4}$. Contudo, esses estudos empregam basicamente soluções-padrão diluídas dos elementos e acidez livre muito diferente daquela empregada na abertura dos minérios de Ti pelas rotas convencionais. Para uma aplicabilidade da proposta de ELL, é preciso estudos de caso com amostras reais (minérios tratados com ácidos minerais e processamento da lixívia ácida obtida).

Em soluções orgânicas as moléculas do D2EHPA apresentamse em equilíbrio químico entre as formas monômera (HD) e dímera $\left(\mathrm{H}_{2} \mathrm{D}_{2}\right)^{: 32}$

$2 \mathrm{HD}_{(\mathrm{o})} \leftrightarrow \mathrm{H}_{2} \mathrm{D}_{2(\mathrm{o})}$

As espécies químicas formadas entre o Fe(III) e o D2EHPA dependem basicamente da concentração de Fe(III), da concentração de D2EHPA livre, da acidez livre e da força iônica da fase aquosa. A abordagem mais simples é a formação de complexos entre o Fe(III) e o D2EHPA dimerizado na razão 1:3. ${ }^{33-35}$

$\mathrm{Fe}^{3+}{ }_{(\mathrm{aq})}+3 \mathrm{H}_{2} \mathrm{D}_{2(\mathrm{o})} \leftrightarrow \mathrm{Fe}\left(\mathrm{HD}_{2}\right)_{3(\mathrm{o})}+3 \mathrm{H}^{+}{ }_{\text {(aq) }}$

O Ti(IV) possui comportamento similar ao Fe(III) no processo de distribuição, porém com efeitos mais pronunciados, devido à relação carga/raio iônico ser maior. ${ }^{31,35} \mathrm{O}$ Ti(IV) em meio aquoso tende a hidrolisar originando oxocátions como o íon titanila, $\mathrm{TiO}^{2+}$, e produtos poliméricos, quando a acidez do meio decresce. Sais básicos pouco solúveis começam a formar-se em $\mathrm{pH}=1,9$. A hidrólise é praticamente completa em $\mathrm{pH}=4,0 .{ }^{23-29}$

$\mathrm{O}$ íon $\mathrm{TiO}^{2+}$ leva à formação de complexos com o D2EHPA na razão $1: 2.25,34$

$\mathrm{TiO}^{2+}{ }_{(\mathrm{aq})}+2 \mathrm{H}_{2} \mathrm{D}_{2(\mathrm{o})} \leftrightarrow \mathrm{TiO}\left(\mathrm{HD}_{2}\right)_{2(\mathrm{o})}+2 \mathrm{H}_{(\text {(aq })}^{+}$

$\mathrm{Fe}^{3+}$ e $\mathrm{TiO}^{2+}$ são as principais espécies químicas de $\mathrm{Fe}(\mathrm{III})$ e Ti(IV) encontradas em meio aquoso.

O presente trabalho foi desenvolvido visando a separação e purificação do $\mathrm{Fe}(\mathrm{III})$ e Ti(IV) oriundos de soluções da abertura sulfúrica da ilmenita, empregando soluções orgânicas de D2EHPA. A principal característica foi a utilização de soluções com altas concentrações dos elementos envolvidos e elevada acidez livre dessas soluções, em contraste com os estudos da literatura, conduzidos em condições experimentais muito diferentes. Buscou-se verificar a aplicabilidade da ELL a uma situação que corresponde à realidade quando se processam minérios de Ti, com a expectativa de menor geração de resíduos de processo em relação às metodologias convencionais de separação Fe-Ti.

\section{PARTE EXPERIMENTAL}

\section{Matéria-prima de $\mathbf{T i}$}

Foram empregadas amostras de ilmenita oriundas do beneficiamento das areias monazíticas de depósitos aluvionares marinhos da região litorânea de Buena, $2^{\circ}$ Distrito de São Francisco de Itabapoana (RJ), pela INB. A ilmenita beneficiada tinha granulometria aproximada $\phi=0,149 \mathrm{~mm}$. A difração de raios-x acusou estrutura do tipo $\beta$ $\mathrm{TiO}_{2}$ (anatásio). A fluorescência de raios-x mostrou que, apesar de ser considerada um material industrial, a ilmenita tinha pureza acima de $98 \% \mathrm{~m} / \mathrm{m}$, apresentando pequenos teores de $\mathrm{Cr}$, Th, lantanídios, U, V e $\mathrm{Zr}$ (em torno de $0,1 \% \mathrm{~m} / \mathrm{m}$ ), oriundos de outros minerais da areia monazítica. As amostras foram pulverizadas em moinho de rolos até diâmetro de partícula $(\phi)<0,074 \mathrm{~mm}$, com o objetivo de aumentar a reatividade no processo de abertura. ${ }^{6,11,31}$

\section{Abertura da ilmenita}

Os experimentos foram realizados em béquer de $250 \mathrm{~mL}$, a $120{ }^{\circ} \mathrm{C}$ por até $2 \mathrm{~h}$, com homogeneização da mistura reacional. A massa de amostra foi fixada em $50 \mathrm{~g}$. As concentrações das soluções de $\mathrm{H}_{2} \mathrm{SO}_{4}$ empregadas foram 7, 13 e $18 \mathrm{~mol} \mathrm{~L}{ }^{1}$. As razões mássicas ilmenita/ $\mathrm{H}_{2} \mathrm{SO}_{4}\left(\mathrm{R}_{\mathrm{M}}\right)$ foram 1:1, 1:2 e 1:3. As condições experimentais acima estão dentro da faixa operacional encontrada para a digestão da ilmenita pelo processo sulfato. ${ }^{4-6,8,10}$

\section{Extração de Ti(IV) por D2EHPA}

Foram utilizadas quatro soluções para a determinação dos coeficientes de distribuição $\left(\mathrm{K}_{\mathrm{D}}\right)$ das espécies químicas de interesse, $\mathrm{H}_{2} \mathrm{SO}_{4}, \mathrm{Fe}(\mathrm{III})$ e Ti(IV): soluções de $\mathrm{H}_{2} \mathrm{SO}_{4}$; soluções de $\mathrm{Fe}_{2}\left(\mathrm{SO}_{4}\right)_{3}$ em $\mathrm{H}_{2} \mathrm{SO}_{4}$; soluções de $\mathrm{TiOSO}_{4}$ em $\mathrm{H}_{2} \mathrm{SO}_{4}$ e, soluções sulfúricas de ilmenita. O objetivo da primeira solução foi determinar a interação do $\mathrm{H}_{2} \mathrm{SO}_{4}$ com a fase orgânica; a segunda e terceira soluções serviram para a determinação dos valores de $\mathrm{K}_{\mathrm{D}}$ para o Fe(III) e Ti(IV) puros em função de suas concentrações e o efeito da variação da acidez livre das soluções aquosas; a quarta solução objetivou verificar os efeitos da presença simultânea de Fe(III) e de Ti(IV) na extração de ambos, em função da acidez livre da solução de abertura da ilmenita (adicionou-se água ou $\mathrm{H}_{2} \mathrm{SO}_{4}$ a essa solução a fim de variar a acidez livre).

As extrações foram efetuadas em funil de separação de 120 $\mathrm{mL}$, onde foram adicionados $50 \mathrm{~mL}$ das soluções aquosas preparadas e $50 \mathrm{~mL}$ da solução orgânica de D2EHPA $\left(1,5 \mathrm{~mol} \mathrm{~L}^{-1}\right)$ em n-dodecano. As fases foram misturadas por agitação mecânica durante $8 \mathrm{~h}$, de forma a garantir que o equilíbrio de distribuição das espécies químicas entre as fases aquosa e orgânica fosse alcançado. As fases foram decantadas e separadas. Foram determinadas as concentrações de $\mathrm{H}_{2} \mathrm{SO}_{4}$, $\mathrm{Fe}$ (III) e Ti(IV) nas fases aquosas originais e após o equilíbrio para cálculo dos $K_{D}$.

A fase orgânica contendo Ti(IV) foi lavada com solução de $\mathrm{H}_{2} \mathrm{SO}_{4} 4,5 \mathrm{~mol} \mathrm{~L}^{-1}$, à temperatura ambiente, com o intuito de remover Fe(III) eventualmente co-extraído ${ }^{33-35}$ (um estágio, relação volumétrica fase aquosa/fase orgânica $[\mathrm{FA} / \mathrm{FO}]=2$ ).

\section{Reextração do Ti}

O Ti(IV) contido na fase orgânica pode ser reextraído por soluções aquosas ácidas contendo íons $\mathrm{F}^{-}$, com formação de fluorcomplexos aniônicos: $:^{1,4,36}$

$\mathrm{TiO}\left(\mathrm{HD}_{2}\right)_{2(\mathrm{o})}+6 \mathrm{~F}_{(\mathrm{aq})}^{-}+4 \mathrm{H}_{(\mathrm{aq})}^{+} \rightarrow\left[\mathrm{TiF}_{6}\right]_{(\mathrm{aq})}^{2-}+\mathrm{H}_{2} \mathrm{O}_{(\mathrm{l})}+2 \mathrm{H}_{2} \mathrm{D}_{2(\mathrm{o})}$

Empregou-se solução aquosa de $\mathrm{NH}_{4} \mathrm{HF}_{2}\left(50 \mathrm{~g} \mathrm{~L}^{-1}, \mathrm{pH} 4,5\right) \mathrm{e}$ uma razão $\mathrm{FA} / \mathrm{FO}=1$ (vol./vol.). A reextração foi feita à temperatura ambiente em frasco de polietileno, sendo o tempo de contato estipulado em $10 \mathrm{~min}$. O processo foi repetido mais duas vezes. As três fases aquosas foram reunidas e homogeneizadas.

O fluorcomplexo formado pode ser decomposto pela elevação do $\mathrm{pH}(\sim 10)$ pela adição de $\mathrm{NH}_{3 \text { (aq) }}$, até a precipitação do hidróxido de titanila (reação 5):1,4,36

$\left[\mathrm{TiF}_{6}\right]_{(\text {aq) }}^{2-}+6 \mathrm{NH}_{3(\mathrm{aq})}+5 \mathrm{H}_{2} \mathrm{O}_{(1)} \rightarrow \mathrm{TiO}(\mathrm{OH})_{2(\mathrm{~s})}+6 \mathrm{NH}_{4} \mathrm{~F}_{(\text {aq })}+2 \mathrm{OH}_{(\text {aq })}^{-}$

O sólido foi lavado com água até ausência de íons $\mathrm{F}^{-}\left(3 \mathrm{~mL} \mathrm{~g}{ }^{-1}\right)$, e calcinado em mufla a $900{ }^{\circ} \mathrm{C}$ :

$\mathrm{TiO}(\mathrm{OH})_{2(\mathrm{~s})} \stackrel{\Delta}{\longrightarrow} \mathrm{TiO}_{2(\mathrm{~s})}+\mathrm{H}_{2} \mathrm{O}_{(\mathrm{v})}$ 
Uma amostra do sólido antes da calcinação foi dissolvida em $\mathrm{H}_{2} \mathrm{SO}_{4} 5 \mathrm{~mol} \mathrm{~L}^{-1}$ para análise química do Ti.

\section{Processamento da fase aquosa ácida contendo $\mathrm{Fe}(\mathrm{III})$}

O rafinado obtido após a extração do Ti foi evaporado até cristalização do sulfato $\mathrm{Fe}_{2}\left(\mathrm{SO}_{4}\right)_{3} \cdot 9 \mathrm{H}_{2} \mathrm{O}$, sendo o liquor ácido residual aproveitado para abertura de novas amostras de ilmenita, ou lavagem da fase orgânica contendo Ti e Fe co-extraído (após adição de $\mathrm{H}_{2} \mathrm{SO}_{4}$ concentrado para ajuste da concentração em cada caso).

As soluções aquosas ácidas obtidas após a lavagem da fase orgânica contendo Ti extraído com $\mathrm{H}_{2} \mathrm{SO}_{4} 4,5 \mathrm{~mol} \mathrm{~L}^{-1}$ eram muito diluídas para que o $\mathrm{Fe}$ fosse isolado, como no caso do rafinado. Por isso, buscou-se reaproveitar essa solução para novas aberturas da ilmenita ou lavagens da fase orgânica com Ti extraído. Para tal, foi feito um estudo da extração do $\mathrm{Fe}(\mathrm{III})$ de soluções aquosas aciduladas com $\mathrm{H}_{2} \mathrm{SO}_{4}$. A proposta foi extrair o metal com D2EHPA (1,5 mol L ${ }^{-1}$ em n-dodecano), numa relação $\mathrm{FA} / \mathrm{FO}=1: 1$ (vol./ vol.), variando-se a acidez livre da solução aquosa (expressa em concentração de $\mathrm{H}_{2} \mathrm{SO}_{4}$ ).

A solução aquosa original foi diluída com $\mathrm{H}_{2} \mathrm{O}$ deionizada, nas proporções volumétricas de 1:1 até 1:5. $25 \mathrm{~mL}$ da solução diluída e $25 \mathrm{~mL}$ da fase orgânica foram colocados em frasco de polietileno, e a mistura foi agitada mecanicamente por $8 \mathrm{~h}$. As fases foram separadas. Nas soluções aquosas antes e após a extração foram determinadas as concentrações de $\mathrm{H}_{2} \mathrm{SO}_{4}$ (acidez livre) e de $\mathrm{Fe}$ (III). $\mathrm{O}$ resultado considerado ideal é obtido ao minimizar a diluição da fase aquosa ácida original.

\section{Reextração e precipitação do Fe}

$\mathrm{O} \mathrm{Fe}(\mathrm{III})$ na fase orgânica pode ser reextraído por soluções aquosas ácidas contendo íons $\mathrm{F}^{-}$, devido à formação de fluorcomplexos aniônicos em meio aquoso, que não são extraídos por soluções orgânicas de D2EHPA:1,4,36

$\mathrm{Fe}^{3+}{ }_{(\mathrm{aq})}+6 \mathrm{~F}_{(\mathrm{aq})}^{-} \rightarrow\left[\mathrm{FeF}_{6}\right]_{(\mathrm{aq})}^{3-}$

$\mathrm{Fe}\left(\mathrm{HD}_{2}\right)_{3(\mathrm{o})}+6 \mathrm{~F}^{-}{ }_{(\mathrm{aq})}+3 \mathrm{H}^{+}{ }_{\text {(aq) }} \rightarrow\left[\mathrm{FeF}_{6}\right]^{3-}{ }_{(\mathrm{aq})}^{3-}+3 \mathrm{H}_{2} \mathrm{D}_{2(\mathrm{o})}$

Não é recomendável o emprego de soluções aquosas neutras ou alcalinas na reextração, devido à possibilidade da extração de cátions como o $\mathrm{NH}_{4(\mathrm{aq})}^{+}$pela fase orgânica, gerando sais (sabões) que formam emulsões estáveis entre as fases, dificultando o processo de separação. ${ }^{31,33}$ A solução de reextração $\left(\mathrm{NH}_{4} \mathrm{HF}_{2}, \mathrm{NH}_{4} \mathrm{~F}\right.$ ou $\mathrm{HF}, 50-100 \mathrm{~g} \mathrm{~L}^{-1}$ ) foi acidulada com $\mathrm{H}_{2} \mathrm{SO}_{4}$ a $\mathrm{pH} 4,5$.

As razões volumétricas entre a fase orgânica e a fase aquosa $\left(R_{F}\right)$ foram variadas $(0,4$ a 8,0$)$ de forma a minimizar o volume de fase aquosa empregado no processo de reextração.

A frascos de polietileno foram adicionadas a fase orgânica e a solução aquosa no valor de $\mathrm{R}_{\mathrm{F}}$ desejado, sendo o volume total final de $50 \mathrm{~mL}$. A mistura foi agitada por $8 \mathrm{~h}$. As fases foram decantadas e separadas. Na fase aquosa determinou-se a concentração de Fe(III).

O fluorcomplexo de $\mathrm{Fe}(\mathrm{III})$ pode ser hidrolisado pela elevação do $\mathrm{pH}(\sim 8)$ pela adição de $\mathrm{NH}_{3 \text { (aq) }}\left(15 \mathrm{~mol} \mathrm{~L}^{-1}\right)$, em porções de 1,00 $\mathrm{mL}$, até a precipitação do hidróxido: ${ }^{1,4,36}$

$\left[\mathrm{FeF}_{6}\right]_{(\mathrm{aq})}^{3-}+6 \mathrm{NH}_{3(\mathrm{aq})}+6 \mathrm{H}_{2} \mathrm{O}_{(1)} \rightarrow \mathrm{Fe}(\mathrm{OH})_{3(\mathrm{~s})}+6 \mathrm{NH}_{4} \mathrm{~F}_{(\mathrm{aq})}+3(\mathrm{OH})_{(\mathrm{aq})}^{-}$

O hidróxido foi lavado com água até ausência de íons $\mathrm{F}^{-}$(4 mL $\left.\mathrm{g}^{-1}\right)$, e depois calcinado $\left(400{ }^{\circ} \mathrm{C}\right)$ para obtenção do óxido:

$2 \mathrm{Fe}(\mathrm{OH})_{3(\mathrm{~s})} \stackrel{\Delta}{\longrightarrow} \mathrm{Fe}_{2} \mathrm{O}_{3(\mathrm{~s})}+3 \mathrm{H}_{2} \mathrm{O}_{(\mathrm{v})}$
Foram determinados o pH e a concentração de Fe(III) remanescente em função dos volumes de $\mathrm{NH}_{3 \text { (aq) }}$ adicionados durante $\mathrm{o}$ processo de precipitação. $\mathrm{O}$ óxido foi dissolvido em $\mathrm{HCl} 5 \mathrm{~mol} \mathrm{~L}^{1}$ para análise química.

\section{Reciclo das soluções aquosas amoniacais contendo $\mathrm{F}^{-}$após precipitação do $\mathrm{Fe}$ (III) e do $\mathrm{Ti}(\mathrm{IV})$}

O foco foi a determinação do número de reusos possíveis da solução de reextração de $\mathrm{Fe}(\mathrm{III})$ da fase orgânica, tomando-se como parâmetro a eficiência de recuperação do metal em comparação com aquela da solução original. Tal prática também foi posteriormente aplicada às soluções amoniacais fluorídricas para o isolamento do Ti.

$\mathrm{O}$ pH da solução amoniacal residual contendo $\mathrm{F}^{-}$foi ajustado para 4,5 com $\mathrm{H}_{2} \mathrm{SO}_{4} 18 \mathrm{~mol} \mathrm{~L}^{-1}$ e contactada com nova solução orgânica contendo $\mathrm{Fe}$ ou Ti, procedendo-se da mesma forma. Foram testados até 4 reciclos das fases aquosas. Através das análises dos teores de Fe ou Ti encontrados, foram monitoradas as eficiências de reextração do metal.

\section{Remoção do íon fluoreto de efluentes finais}

Entende-se por efluente final as águas de lavagem dos precipitados de Ti e de Fe. Segundo a resolução no 357/05 ${ }^{37}$ do Conselho Nacional do Meio Ambiente (CONAMA), a concentração máxima de $\mathrm{F}^{-}$em efluentes é de $10 \mathrm{mg} \mathrm{L}^{-1}$. Assim, uma meta primordial para uma boa gestão de um processo químico é a remoção do íon $\mathrm{F}^{-}$ dessas águas de lavagem pela formação de $\mathrm{CaF}_{2}$ a partir da adição de $\mathrm{CaCl}_{2} 2 \mathrm{H}_{2} \mathrm{O}$ ou $\mathrm{Ca}(\mathrm{OH})_{2}$. O sólido foi adicionado diretamente à solução, à temperatura ambiente (200 rpm), até cessar a precipitação do sólido, que foi filtrado.

\section{Métodos analíticos}

As soluções aquosas de $\mathrm{H}_{2} \mathrm{SO}_{4}$ foram dosadas por meio de titulação de neutralização direta contra $\mathrm{NaOH} 0,10 \mathrm{~mol} \mathrm{~L}^{-1}$, empregando azul de bromotimol como indicador, ou potenciométricas, empregando eletrodo combinado de $\mathrm{Ag} / \mathrm{AgCl}$. No caso das amostras fez-se uma adição prévia de citrato de sódio $0,1 \mathrm{~mol} \mathrm{~L}^{-1}$ (evitar a hidrólise do $\mathrm{Fe}$ e do Ti), seguido de titulação conforme descrito acima.

Para as análises por difração de raios-x (difratômetro Philips PW 1820; fonte de radiação $\mathrm{Cu}-\mathrm{K} \alpha$, varredura angular (2 teta) 12 $70^{\circ}$, com passo de $0,020^{\circ}$ e tempo de $1 \mathrm{~s}$ por passo), as amostras de ilmenita e do produto final de Ti foram moídas e homogeneizadas em gral de ágata (granulometria menor que $0,0044 \mathrm{~mm}$ ). Uma massa de aproximadamente $0,1 \mathrm{~g}$ foi colocada sobre um papel-filtro $(\phi=25$ $\mathrm{mm})$ fixado no centro de um filme de PVC adesivo $(\phi=45 \mathrm{~mm})$ cobrindo-se as amostras com filme de poliéster. Os difratogramas obtidos foram comparados com padrões de ilmenita, anatásio e rutilo. $^{38}$

Para as análises por fluorescência de raios-x (FRX) alíquotas de $200 \mu \mathrm{L}$ de soluções-padrão de Fe e Ti $\left(0,1-1,0 \mathrm{~g} \mathrm{~L}^{-1}\right)$ foram pipetadas em papel de filtro $(\phi=25 \mathrm{~mm})$, secos sob lâmpada de infravermelho, fixadas no centro de um filme de PVC adesivo $(\phi=45 \mathrm{~mm})$, e cobertos por filme de poliéster, permitindo assim a construção das curvas de calibração. A varredura das amostras e padrões foi feita em aparelho Rigaku modelo B3, com detector de $\mathrm{LiF}$ e tubo de Ag. Uma grande vantagem desta análise é a não interferência mútua entre os espectros do Fe e do Ti.

A concentração dos íons $\mathrm{F}^{-}$no efluente final tratado foi determinada através de um eletrodo seletivo para esse íon. 


\section{RESULTADOS E DISCUSSÃO}

\section{Abertura da ilmenita}

Durante o processo de digestão o $\mathrm{Fe}(\mathrm{II})$ presente foi praticamente todo oxidado a Fe(III), devido às condições oxidantes da mistura reacional e às temperaturas elevadas segundo as reações:

$$
\begin{aligned}
& \mathrm{FeTiO}_{3(\mathrm{~s})}+3 \mathrm{H}_{2} \mathrm{SO}_{4(\mathrm{aq})} \stackrel{\Delta}{\longrightarrow} \mathrm{FeSO}_{4(\mathrm{aq})}+\mathrm{Ti}\left(\mathrm{SO}_{4}\right)_{2(\mathrm{aq})}+3 \mathrm{H}_{2} \mathrm{O}_{(1)}(11) \\
& 2 \mathrm{FeSO}_{4(\mathrm{aq})}+\mathrm{H}_{2} \mathrm{SO}_{4(\mathrm{aq})}+1 / 2 \mathrm{O}_{2(\mathrm{~g})} \stackrel{\Delta}{\longrightarrow} \mathrm{Fe}_{2}\left(\mathrm{SO}_{4}\right)_{3(\mathrm{aq})}+\mathrm{H}_{2} \mathrm{O}_{(1)}
\end{aligned}
$$

A abertura da ilmenita com $\mathrm{H}_{2} \mathrm{SO}_{4} 7 \mathrm{~mol} \mathrm{~L}^{-1}$, mesmo com razão mássica $\mathrm{R}_{\mathrm{M}}=1: 3$ e tempo de digestão prolongado (maior que $2 \mathrm{~h}$ ), não forneceu resultados satisfatórios, com solubilizações inferiores a $25 \%$ da massa original.

A abertura com $\mathrm{H}_{2} \mathrm{SO}_{4} 18 \mathrm{~mol} \mathrm{~L}^{-1}, \mathrm{R}_{\mathrm{M}}=1: 2$ ou 1:3, e tempo de digestão em torno de $1,5 \mathrm{~h}$, apresentou uma solubilização do mineral entre 85 e $90 \% \mathrm{~m} / \mathrm{m}$. Todavia, a mistura reacional tornou-se progressivamente mais viscosa, dificultando a preparação para as etapas seguintes. Para mesmos valores de $\mathrm{R}_{\mathrm{M}}$ e tempo de digestão, a abertura com $\mathrm{H}_{2} \mathrm{SO}_{4} 13 \mathrm{~mol} \mathrm{~L}^{-1}$ apresentou eficiência um pouco inferior àquela com $\mathrm{H}_{2} \mathrm{SO}_{4} 18 \mathrm{~mol} \mathrm{~L}^{-1}$ (Tabela 1), mas a mistura reacional final apresentou-se fluida o bastante para permitir sua manipulação posterior. A partir desses resultados, foi estabelecido o processo de digestão otimizado com $\mathrm{R}_{\mathrm{M}}=1: 3$ entre ilmenita e $\mathrm{H}_{2} \mathrm{SO}_{4}$, sendo o tempo médio gasto de $1,5 \mathrm{~h}$, e eficiência de abertura próxima a $90 \% \mathrm{~m} / \mathrm{m}$, comparável a outros estudos da literatura. ${ }^{6,810,39}$ Um tempo de digestão maior não contribuiu significativamente para o aumento da eficiência da abertura.

Após o resfriamento da mistura reacional, esta foi tratada com $\mathrm{H}_{2} \mathrm{O}$ à temperatura ambiente (1:1 vol./vol.), evitando o superaquecimento da solução para não ocasionar hidrólise parcial do Ti(IV), decantada e filtrada, gerando soluções castanhas contendo concentrações médias de $(21,1 \pm 1,0) \mathrm{g} \mathrm{Fe} \mathrm{L}^{-1},(25,5 \pm 0,7) \mathrm{g} \mathrm{Ti} \mathrm{L}{ }^{-1} \mathrm{e}(2,5$ $\pm 0,2) \mathrm{mol} \mathrm{L}^{-1}$ de $\mathrm{H}_{2} \mathrm{SO}_{4}$.

Tabela 1. Abertura da ilmenita por $\mathrm{H}_{2} \mathrm{SO}_{4}$ sob várias condições experimentais $\left(120^{\circ} \mathrm{C}\right)$

\begin{tabular}{cccc}
\hline$\left[\mathrm{H}_{2} \mathrm{SO}_{4}\right]\left(\mathrm{mol} \mathrm{L}^{-1}\right)$ & $\mathrm{R}_{\mathrm{M}}{ }^{*}$ & Tempo de digestão (h) & $\begin{array}{r}\% \mathrm{~m} / \mathrm{m} \\
\text { dissolvido }\end{array}$ \\
\hline 13 & $1: 1$ & 1,0 & 41,2 \\
& & 1,5 & 47,0 \\
& $1: 2$ & 1,0 & 50,5 \\
& & 1,5 & 60,6 \\
& & 2,0 & 73,0 \\
& $1: 3$ & 1,0 & 75,7 \\
& & 1,5 & 79,4 \\
& & 2,0 & 87,3 \\
& $1: 1$ & 1,0 & 89,0 \\
& & 1,5 & 46,5 \\
& & 2,0 & 58,1 \\
& $1: 2$ & 1,0 & 60,1 \\
& & 1,5 & 67,2 \\
& & 2,0 & 77,2 \\
& $1: 3$ & 1,0 & 81,4 \\
& & 2,0 & 81,0 \\
& & & 89,8 \\
& &
\end{tabular}

* razão mássica ilmenita/ $\mathrm{H}_{2} \mathrm{SO}_{4}$

\section{Determinação dos coeficientes de distribuição $\left(K_{\mathrm{D}}\right)$}

$\mathrm{H}_{2} \mathrm{SO}_{4}$

Soluções aquosas puras de $\mathrm{H}_{2} \mathrm{SO}_{4}$ não sofreram extração significativa pela fase orgânica contendo D2EHPA, ou seja, o coeficiente de distribuição do ácido é próximo a zero.

$\mathrm{Fe}(I I I)$

Os valores de $\mathrm{K}_{\mathrm{D}}$ para este íon atingiram valores em torno de 14 , em $\mathrm{H}_{2} \mathrm{SO}_{4}$ aproximadamente $0,25 \mathrm{~mol} \mathrm{~L}^{-1}$, diminuindo progressivamente até um valor mínimo $\mathrm{K}_{\mathrm{D}} \cong 2$ em concentrações do ácido aproximadamente iguais a $2,5 \mathrm{~mol} \mathrm{~L}^{-1}$, descrevendo um comportamento típico da extração por quelação do cátion (Figura 1). ${ }^{1,33,36,40}$ Não houve contração ou dilatação das fases orgânica e aquosa nos experimentos.

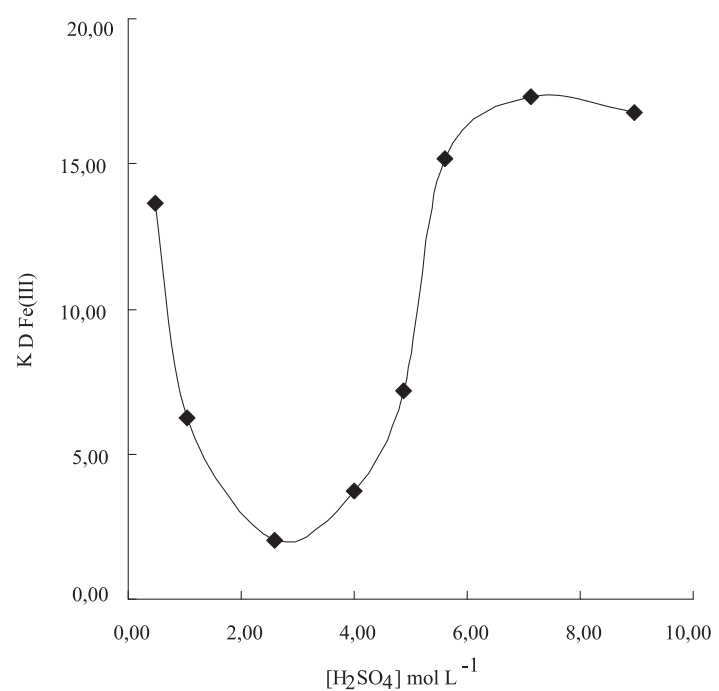

Figura 1. Coeficientes de distribuição para Fe(III) em função da concentração de $\mathrm{H}_{2} \mathrm{SO}_{4}$ na fase aquosa

Nas soluções com concentrações do ácido superiores a 2,5 mol $\mathrm{L}^{-1}$ houve um aumento progressivo dos valores de $\mathrm{K}_{\mathrm{D}}$ devido aos efeitos salino e Donan em soluções aquosas com alta concentração de eletrólitos, ${ }^{1,33}$ até valores máximos da ordem de 17 em $\mathrm{H}_{2} \mathrm{SO}_{4}$ 7,0 mol $\mathrm{L}^{-1}$, região a partir da qual $\mathrm{K}_{\mathrm{D}}$ permaneceu praticamente constante, até o limite da concentração operacional (quando a fase orgânica começa a ser degradada pelo ácido concentrado). $\mathrm{O}$ perfil do $\mathrm{K}_{\mathrm{D}}$ em baixa acidez $\left(<1 \mathrm{~mol} \mathrm{~L}^{-1}\right)$ concorda com os dados da literatura. . $_{\mathrm{D}}^{31,33}$

$\operatorname{Ti}(I V)$

Os valores de $\mathrm{K}_{\mathrm{D}}$ atingiram valores em torno de 5 em $\mathrm{H}_{2} \mathrm{SO}_{4}$ aproximadamente $1,2 \mathrm{~mol} \mathrm{~L}^{-1}$, diminuindo progressivamente até um valor mínimo $\cong 0,5$ em concentrações em torno de $2,5 \mathrm{~mol} \mathrm{~L}^{-1}$, descrevendo um comportamento típico da extração por quelação do cátion (Figura 2). ${ }^{29,33,34}$ Nas soluções com concentração superior a $2,5 \mathrm{~mol} \mathrm{~L}^{-1}$ ocorreu um aumento progressivo dos valores de $\mathrm{K}_{\mathrm{D}}$ devido aos efeitos salino e Donan, ${ }^{25,26,30}$ mais pronunciados que no caso do $\mathrm{Fe}(\mathrm{III})$, até cerca de 70 numa concentração de $\mathrm{H}_{2} \mathrm{SO}_{4}$ aproximadamente igual a $11,8 \mathrm{~mol} \mathrm{~L}^{-1}$. Não houve contração ou dilatação das fases orgânica e aquosa nos experimentos. Os valores obtidos em baixa acidez livre $(<$ $2 \mathrm{~mol} \mathrm{~L}^{-1}$ ) concordam com os dados da literatura. ${ }^{29,31,33} \mathrm{O}$ trabalho nessas condições é dificultado pela forte hidrólise do Ti(IV), levado à precipitação parcial ou mesmo total do metal. ${ }^{23-29}$

$\mathrm{Fe}(I I I)+\mathrm{Ti}(\mathrm{IV})$

Nas extrações realizadas em soluções sulfúricas contendo Fe(III) 


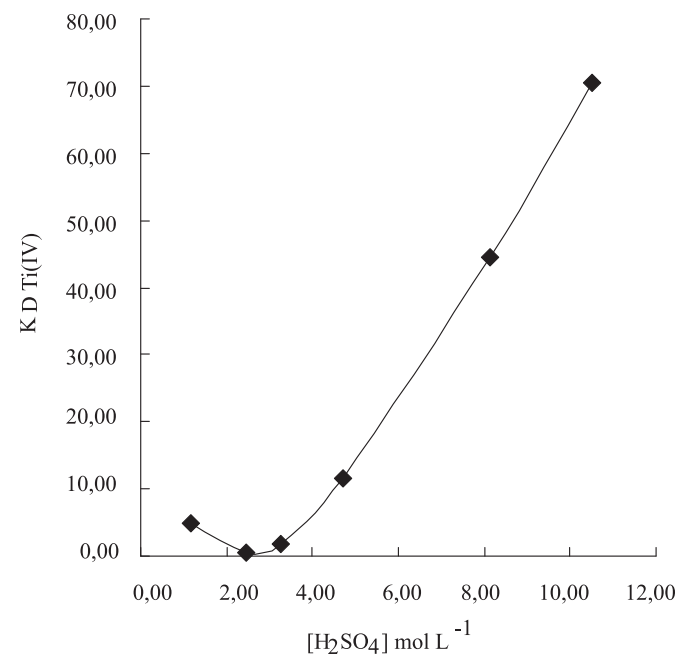

Figura 2. Coeficientes de distribuição para o Ti(IV) em função da concentração de $\mathrm{H}_{2} \mathrm{SO}_{4}$ na fase aquosa

e Ti(IV), após a abertura da ilmenita, as espécies químicas comportaram-se de maneira diversa de quando foram extraídas em separado. $\mathrm{O} \mathrm{K}_{\mathrm{D}}$ do $\mathrm{Ti}(\mathrm{IV})$ aumentou na presença de $\mathrm{Fe}$ (III) (Tabela 2). Em contrapartida, o $\mathrm{K}_{\mathrm{D}}$ do $\mathrm{Fe}(\mathrm{III})$ diminuiu, mantendo este elemento preferencialmente na fase aquosa.

$\mathrm{Na}$ extração de soluções aquosas contendo concomitantemente ambas as espécies químicas por soluções orgânicas de D2EHPA, o equilíbrio termodinâmico da partição do Ti(IV) não é somente favorecido em relação ao do $\mathrm{Fe}(\mathrm{III})$, como aquele é preferencialmente extraído em detrimento do $\mathrm{Fe}(\mathrm{III}),{ }^{23-31}$ tornando este efeito crítico em soluções aquosas com acidez livre baixa $\left(<3 \mathrm{~mol} \mathrm{~L}^{-1}\right)$ e conforme aproxima-se a concentração de saturação na fase orgânica das espécies extraídas..$^{24,27-29}$

Nestas condições, as próprias espécies químicas do Fe(III) associadas também aos íons $\mathrm{SO}_{4}^{2-}$ na fase aquosa contribuem para um aumento da força iônica do meio aquoso. Esse efeito contribui para um maior deslocamento do equilíbrio na partição das espécies de Ti(IV) no sentido de aumentar a extração das mesmas pela fase orgânica ${ }^{25,26,30}$ (efeito Donan).

Em relação à solução de abertura da ilmenita $\left(\mathrm{H}_{2} \mathrm{SO}_{4} 2,5 \mathrm{~mol}\right.$ $\mathrm{L}^{-1}$ ), em dois estágios (Tabela 2), 99,4 $\pm 0,3 \% \mathrm{~m} / \mathrm{m}$ do Ti foi extraído, enquanto que o Fe foi extraído em $15,5 \pm 1,2 \% \mathrm{~m} / \mathrm{m}$.

A reextração desse $\mathrm{Fe}$ com $\mathrm{H}_{2} \mathrm{SO}_{4} 4,5$ mol L-1 removeu 99,4 \pm $0,7 \% \mathrm{~m} / \mathrm{m}$ do metal da fase orgânica, com uma reextração inferior a $1 \% \mathrm{~m} / \mathrm{m}$ do Ti, em conformidade com os dados da Tabela 2.

\section{Reextração e recuperação do Ti(IV) da fase orgânica}

O Ti(IV) foi recuperado em $99,4 \pm 0,3 \% \mathrm{~m} / \mathrm{m}$ da fase orgânica pela solução de $\mathrm{NH}_{4} \mathrm{HF}_{2} 50 \mathrm{~g} \mathrm{~L}^{-1}$, bem como precipitado em mais de $99,9 \% \mathrm{~m} / \mathrm{m}$ após ajuste do $\mathrm{pH}$ em 10 . A análise do produto calcinado a $900{ }^{\circ} \mathrm{C}$ por DRX e FRX mostrou que o $\mathrm{TiO}_{2}$ obtido tinha pureza superior a $99 \% \mathrm{~m} / \mathrm{m}$ e apresentava estrutura cristalina correspondente ao anatásio $\left(\beta-\mathrm{TiO}_{2}\right)$.

\section{Recuperação do $\mathrm{H}_{2} \mathrm{SO}_{4}$ dos efluentes contendo $\mathrm{Fe}(\mathrm{III})$}

Determinação da acidez livre máxima para extração do $\mathrm{Fe}(\mathrm{III})$

De acordo com os dados da Figura 3, a concentração máxima de $\mathrm{H}_{2} \mathrm{SO}_{4}$ para que os valores de $\mathrm{K}_{\mathrm{D}}$ para $\mathrm{Fe}(\mathrm{III})$ sejam elevados (da ordem de $10^{3}$ ), na extração do metal com D2EHPA, não deve ultrapassar 1,2 mol $\mathrm{L}^{-1}$. No caso do Fe removido após a lavagem da fase
Tabela 2. Coeficientes de distribuição $\left(K_{\mathrm{D}}\right)$ e fatores de separação ( $\beta$ ) de soluções aquosas sulfúricas contendo Fe(III) e Ti(IV)

\begin{tabular}{lccc}
\hline$\left[\mathrm{H}_{2} \mathrm{SO}_{4}\right]_{\mathrm{eq}}\left(\mathrm{mol} \mathrm{L}^{-1}\right)$ & $\mathrm{K}_{\mathrm{D}} \mathrm{Fe}(\mathrm{III})$ & $\mathrm{K}_{\mathrm{D}} \mathrm{Ti}(\mathrm{III})$ & $\beta_{(\mathrm{Ti} / \mathrm{Fe})}$ \\
\hline $3,0 \times 10^{-2}$ & 80,91 & $11.254,00$ & 139,10 \\
$4,1 \times 10^{-1}$ & 5,48 & 759,18 & 138,44 \\
$6,4 \times 10^{-1}$ & 3,79 & 515,17 & 135,93 \\
$6,8 \times 10^{-1}$ & 3,56 & 416,26 & 116,94 \\
$8,4 \times 10^{-1}$ & 2,09 & 144,51 & 69,01 \\
$9,6 \times 10^{-1}$ & 1,16 & 81,61 & 70,50 \\
1,20 & 1,06 & 68,90 & 65,00 \\
1,54 & 0,65 & 42,84 & 65,90 \\
1,80 & 0,51 & 27,60 & 54,12 \\
2,15 & 0,33 & 21,84 & 66,18 \\
2,55 & 0,38 & 26,09 & 68,66 \\
3,04 & 0,58 & 28,55 & 49,22 \\
3,63 & 0,67 & 30,60 & 45,67 \\
4,15 & 0,86 & 31,23 & 36,27 \\
4,95 & 0,99 & 39,49 & 39,89 \\
5,58 & 1,80 & 45,16 & 25,11 \\
6,88 & 1,96 & 57,15 & 29,17 \\
8,00 & 3,49 & 73,24 & 21,01 \\
9,35 & 3,69 & 97,34 & 26,39 \\
11,88 & 12,32 & 102,69 & 8,33 \\
\hline
\end{tabular}

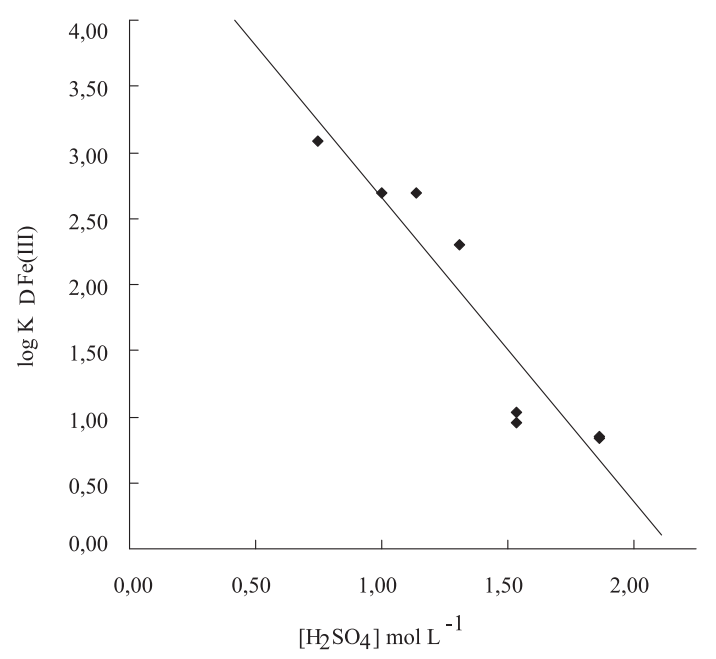

Figura 3. Valores de $\log K_{D \text { Fe(III) }}$ em função da acidez livre da solução aquosa

orgânica contendo Ti, a diluição necessária é 1:4 (a concentração inicial do ácido era $4,5 \mathrm{~mol} \mathrm{~L}^{-1}$ ). Mais de $99,5 \% \mathrm{~m} / \mathrm{m}$ do metal foi extraído em um único estágio nessas condições.

Testes com amostras do rafinado indicam que diluições próximas a 1:2 (a concentração original do ácido nesta solução era da ordem de 2,5 $\mathrm{mol} \mathrm{L}^{-1}$ ) permitiram obter o mesmo desempenho, evitando-se a evaporação para cristalização do sulfato. Para reuso da solução ácida, é preciso adicionar $\mathrm{H}_{2} \mathrm{SO}_{4}$ concentrado até ajuste da concentração a $13 \mathrm{~mol} \mathrm{~L}^{-1}$ (abertura da ilmenita) ou remoção do Fe co-extraído com o Ti $\left(4,5 \mathrm{~mol} \mathrm{~L}^{-1}\right)$.

\section{Reextração e recuperação do $\mathrm{Fe}(\mathrm{III})$ da fase orgânica}

Nas reextrações efetuadas com soluções aquosas de $\mathrm{NH}_{4} \mathrm{HF}_{2} 50 \mathrm{~g} \mathrm{~L}^{-1}$, empregando $\mathrm{R}_{\mathrm{F}} \leq 0,5$, o $\mathrm{Fe}$ (III) foi recuperado com eficiência superior a $90 \% \mathrm{~m} / \mathrm{m}$ (Tabela 3). HF e $\mathrm{NH}_{4} \mathrm{~F}$ tiveram desempenhos inferiores, na mesma concentração e $\mathrm{R}_{\mathrm{F}}$. Para soluções de $\mathrm{NH}_{4} \mathrm{HF}_{2} 100 \mathrm{~g} \mathrm{~L}^{-1}$ empregando $\mathrm{R}_{\mathrm{F}}$ 2,0, o Fe(III) foi recuperado em mais de $95 \% \mathrm{~m} / \mathrm{m}$. O inconveniente do emprego de solu- 
ções de $\mathrm{NH}_{4} \mathrm{HF}_{2}$ de maior concentração é a formação do composto $\left(\mathrm{NH}_{4}\right)_{3}\left[\mathrm{FeF}_{6}\right]$, que possui baixa solubilidade, ${ }^{1,36}$ formando soluções aquosas metaestáveis que tendem a precipitar com o tempo.

Tabela 3. Determinação das $\mathrm{K}_{\mathrm{D} \text { Fe(III) }}$ e $\mathrm{R}_{\mathrm{F}}$ na reextração do $\mathrm{Fe}(\mathrm{III})$ da fase orgânica

\begin{tabular}{lcc}
\hline Agente de extração & $\mathrm{R}_{\mathrm{F}}$ & $\% \mathrm{~m} / \mathrm{m}$ Fe extraído \\
\hline $\mathrm{NH}_{4} \mathrm{~F}\left(50 \mathrm{~g} \mathrm{~L}^{-1}\right)$ & 0,50 & $65,1 \pm 2,1$ \\
& 1,00 & $60,2 \pm 2,2$ \\
$\mathrm{HF}\left(50 \mathrm{~g} \mathrm{~L}^{-1}\right)$ & 2,00 & $31,8 \pm 1,4$ \\
& 1,00 & $73,1 \pm 2,7$ \\
& 2,00 & $46,6 \pm 1,5$ \\
$\mathrm{NH}_{4} \mathrm{HF}_{2}\left(50 \mathrm{~g} \mathrm{~L}^{-1}\right)$ & 4,00 & $27,5 \pm 1,1$ \\
& 0,40 & $96,3 \pm 2,4$ \\
& 0,50 & $92,9 \pm 2,4$ \\
& 1,00 & $74,4 \pm 1,7$ \\
& 2,00 & $59,7 \pm 0,9$ \\
$\mathrm{NH}_{4} \mathrm{HF}_{2}\left(100 \mathrm{~g} \mathrm{~L}^{-1}\right)$ & 4,00 & $49,5 \pm 1,0$ \\
& 1,00 & $>99,5$ \\
& 2,00 & $95,3 \pm 1,8$ \\
& 4,00 & $86,5 \pm 1,4$ \\
& 6,00 & $53,7 \pm 1,2$ \\
& 8,00 & $29,4 \pm 0,5$ \\
\hline
\end{tabular}

Precipitação e recuperação do $\mathrm{Fe}(\mathrm{III})$ das soluções fluorídricas

De acordo com os dados apresentados na Figura 4, a precipitação do $\mathrm{Fe}$ só começou a partir de $\mathrm{pH} 7$, o que indica a necessidade de meio alcalino para a recuperação, conforme a literatura. ${ }^{1,436} \mathrm{O}$ metal precipitou com rendimento superior a $99,9 \% \mathrm{~m} / \mathrm{m}$.

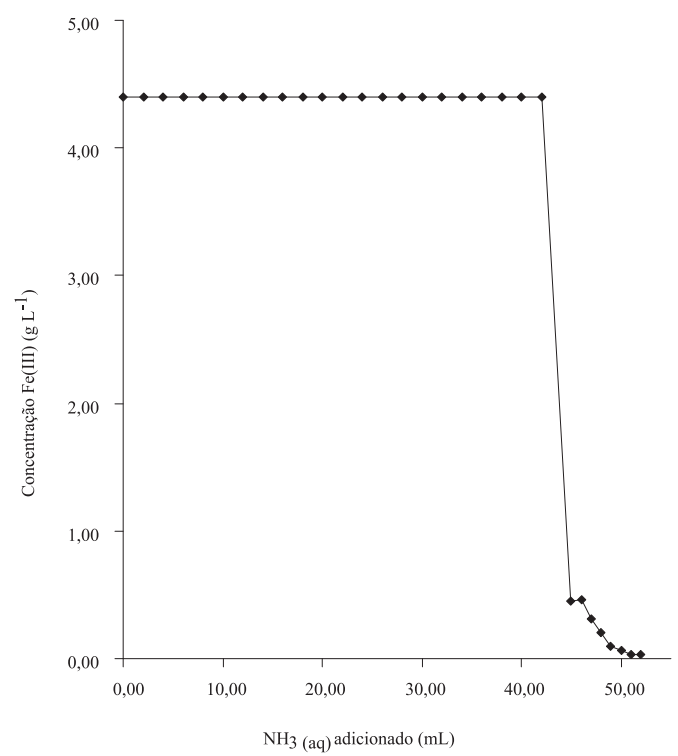

Figura 4. Variação da concentração de $\mathrm{Fe}(\mathrm{III})$ em função do $\mathrm{pH}$ na precipitação do $\mathrm{Fe}(\mathrm{III})$ de soluções fluorídricas

\section{Reciclo das soluções aquosas fluorídricas}

Os resultados apresentados na Tabela 4 indicam uma eficiência comparável da reextração do $\mathrm{Fe}(\mathrm{III})$ e do Ti(IV) no $2^{\circ}$ e $3^{\circ}$ reciclos em relação à reextração original, devido à diminuição da força iônica das soluções pelas diluições, facilitando uma maior extração dos metais. A partir do 4 o reciclo a eficiência de reextração diminuiu significativamente porque as diluições sucessivas reduziram a concentração dos íons $\mathrm{F}^{-}$(Tabela 4). Nessas circunstânci- as, pode-se adicionar $\mathrm{HF}$ a fim de ajustar a concentração do íon $\mathrm{F}$ ao valor desejado, ou então precipitá-lo como $\mathrm{CaF}_{2}$.

Tabela 4. Recuperação de Fe(III) e de Ti(IV) da fase orgânica em função do número de reciclos da solução de $\mathrm{NH}_{4} \mathrm{HF}_{2}{ }^{*}$

\begin{tabular}{lcc}
\hline Amostra & $\% \mathrm{~m} / \mathrm{m} \mathrm{Fe}$ recuperado & $\% \mathrm{~m} / \mathrm{m}$ Ti recuperado \\
\hline $\mathrm{F}_{\text {(aq) }}$ Original & $95,3 \pm 1,8$ & $99,4 \pm 0,3$ \\
$1^{0}$ Reciclo & $86,9 \pm 1,3$ & $90,9 \pm 0,8$ \\
$2^{0}$ Reciclo & $91,2 \pm 2,0$ & $95,2 \pm 1,1$ \\
$3^{0}$ Reciclo & $94,7 \pm 1,7$ & $96,2 \pm 1,1$ \\
$4^{0}$ Reciclo & $74,9 \pm 1,4^{* *}$ & $81,5 \pm 1,0^{* * *}$ \\
\hline
\end{tabular}

$* \mathrm{R}_{\mathrm{F}}=2,0$ (vol./vol.), $100 \mathrm{~g} \mathrm{~L}^{-1} \mathrm{NH}_{4} \mathrm{HF}_{2}, \mathrm{pH} 4,5$ (Fe); FA/FO = 1 (vol./ vol.), $50 \mathrm{~g} \mathrm{~L}^{-1} \mathrm{NH}_{4} \mathrm{HF}_{2}, \mathrm{pH} 4,5$ (Ti); ** Após o $4^{\circ}$ reciclo, a concentração de $\mathrm{F}^{-}\left(\right.$como $\left.\mathrm{NH}_{4} \mathrm{HF}_{2}\right)$ era $(87,5 \pm 2,0) \mathrm{g} \mathrm{L}^{-1}$; *** Após o $4^{\circ}$ reciclo, a concentração de $\mathrm{F}^{-}\left(\right.$como $\left.\mathrm{NH}_{4} \mathrm{HF}_{2}\right)$ era $(40,6 \pm 1,0) \mathrm{g} \mathrm{L}^{-1}$.

O reciclo das soluções fluorídricas é fundamental para que haja uma menor geração de resíduos de processo por massa de ilmenita processada. Além disso, tanto a extração como a reextração do Ti e do Fe são feitas em condições experimentais bem mais brandas que os processos convencionais relatados na literatura. ${ }^{1,4,8,18-22}$ Contudo, as soluções de reextração contêm o íon $\mathrm{F}^{-}$, grave poluente ambiental, exigindo manipulação cuidadosa, mesmo levando-se em contra que a faixa de $\mathrm{pH}$ de trabalho $(4,5-10)$ está fora da condição considerada corrosiva ( $\mathrm{pH}<2$ ou $>12,5)$, e que o processo é conduzido à temperatura ambiente. $\mathrm{O}$ reciclo dessas soluções não apenas reduz o consumo de insumos, mas também representa um cuidado ambiental.

\section{Remoção do íon $F^{-}$dos efluentes finais}

A concentração do íon $\mathrm{F}^{-}$após tratamento com sais de $\mathrm{Ca}^{2+}$ é de $1,2 \pm 0,2 \mathrm{mg} \mathrm{L}^{-1}$, enquadrando o mesmo para descarte em corpo receptor, dentro das normas da Resolução 357/05 do CONAMA. ${ }^{37}$ Para cada kg de ilmenita processada, obteve-se cerca de $30 \mathrm{~g}$ de $\mathrm{CaF}_{2}$, que podem ser dispostos em aterros classe I para resíduos perigosos.

\section{CONCLUSÕES}

O processamento da solução sulfúrica de ilmenita através da extração líquido-líquido com D2EHPA em n-dodecano, seguido de reextração por soluções aquosas contendo íons $\mathrm{F}^{-}$, mostrou-se eficiente para separar o Ti do Fe contidos na amostra estudada. Uma das principais vantagens é a possibilidade de reciclo dos insumos empregados no processo, acarretando numa diminuição significativa da geração de resíduos finais de processo.

Devido aos grandes valores dos fatores de separação entre os elementos $\left(\beta_{\mathrm{TiFe}}\right)$ no sistema proposto, é possível utilizar um número reduzido de estágios de extração, obtendo-se produtos finais de elevada pureza. A extração preferencial do Ti sobre o Fe é um fator positivo que viabiliza o processo de separação e purificação dos elementos em condições experimentais brandas.

\section{MATERIAL SUPLEMENTAR}

O material suplementar, disponível gratuitamente em http:// quimicanova.sbq.org.br na forma de arquivo PDF, apresenta o fluxograma detalhado de todas as etapas de separação $\mathrm{Fe}-\mathrm{Ti}$ a partir da ilmenita tratada com $\mathrm{H}_{2} \mathrm{SO}_{4}$ (Figura $1 \mathrm{~S}$ ). 


\section{REFERÊNCIAS}

1. Busev, A. I.; Triptsova, V. G.; Ivanov, V. M.; Analytical Chemistry of Rare Elements, Mir: Moscow, 1981.

2. http://www.dnpm.gov.br/dnpm_legis/suma2000, acessada em Julho 2006.

3. http://www.mineral.usgs.gov, acessada em Julho 2005.

4. Othmer, D. F.; Encyclopedia of Chemical Technology, $3^{\text {rd }}$ ed., John Wiley: Nova Iorque, 1983.

5. El-Hazek, N.; Lasheen, T. A.; El-Sheikh, R.; Zaki, S. A.; Hydrometallurgy 2007, 87, 45 .

6. Liang, B.; Li, C.; Zhang, C.; Zhang, Y.; Hydrometallurgy 2005, 76, 173.

7. $\mathrm{TiO}_{2}$ Ceramic Grade, Ceramic Industry: Troy (Michigam, EUA), 1996, vol. 146.

8. Liu, Y.; Qi, T.; Chu, J.; Tong, O.; Zhang, Y.; Int. J. Min. Proc. 2006, 81, 79.

9. Malati, M. A.; Environ. Technol. 1995, 15, 1093.

10. Sarker, M. K.; Rashid, A. K. M. B.; Kurny, A. S. W.; Int. J. Min. Proc. 2006, 80, 223.

11. Li, C.; Liang, B.; Chen, S. P.; Hydrometallurgy 2006, 82, 93.

12. Lanyon, M. R.; Lwin, T.; Merritt, R. R.; Hydrometallurgy 1999, 49, 299.

13. Olanipekun, E.; Hydrometallurgy 1999, 53, 1.

14. Ogasawara, T.; Araújo, R. V. V.; Hydrometallurgy 2000, 56, 203.

15. Dyk, J. P.; Vegter, N. M.; Pistorius, P. C.; Hydrometallurgy 2002, 65, 31.

16. Amer, A. M.; Hydrometallurgy 2002, 67, 125.

17. Mahmoud, M. H. H.; Afifi, A. A. I.; Ibrahim, I. A.; Hydrometallurgy 2004, $73,99$.

18. Liberti, A.; Ciavatta, L.; J. Inorg. Nucl. Chem. 1958, 8, 365.

19. Liberti, A.; Chiantella, V.; Corigliano, F.; J. Inorg. Nucl. Chem. 1963, 25, 415.

20. Baes Jr., C. F.; Mesmer, R. E.; The Hydrolysis of Cations, John Wiley \& Sons: Nova Iorque, 1976, cap. 8.
21. Babko, A. K.; Mazurenko, E. A.; Nabivanets, B. I.; Russ. J. Inorg. Chem. 1969, 14, 1091.

22. Babko, A. K.; Gridchina, G. I.; Nabivanets, B. I.; Russ. J. Inorg. Chem. 1962, 7, 210.

23. Biswas, R. K.; Begum, D. A.; Hydrometallurgy 1998, 49, 263.

24. Biswas, R. K.; Begum, D. A.; Hydrometallurgy 2000, 55, 57.

25. Biswas, R. K.; Zaman, M. R.; Islam, M. N.; Hydrometallurgy 2002, 63, 159.

26. Singh, R. K.; Dhadke, P. M.; J. Serb. Chem. Soc. 2002, 61, 507.

27. Chaudry, M. A.; Malik M. T.; Ahmad, M.; J. Radioanal. Nucl. Chem. 1992, $157,143$.

28. Io, K.; Takahashi, K.; Takeuchi, H.; Solvent Extr. Ion Exch. 1991, 9, 27.

29. Phalke, P. N.; Sherikar, A. V.; Dhadke, P. M.; Indian J. Chem., Sect. A.: Inorg., Bio-inorg., Phys., Theor. Anal. Chem. 1997, 36, 446.

30. Islam, M. F; Biswas, R. K.; J. Inorg. Nucl. Chem. 1978, 40, 559.

31. Sole, K. C.; Hydrometallurgy 1999, 51, 239.

32. Dyrssen, D.; Acta Chem. Scand. 1957, 11, 1771.

33. Islam, M. F.; Biswas, R. K.; J. Inorg. Nucl. Chem. 1981, 43, 1929.

34. Sato, T.; Nakamura, T.; Anal. Chem. Acta 1975, 76, 401.

35. Baes Jr., C. F.; Baker, H. T.; J. Phys. Chem. 1960, 64, 89.

36. Minczewski, J.; Chwastowska, J.; Dybczyñski, R.; Separation and Preconcentration Methods in Inorganic Analysis, John Wiley \& Sons: Chichester, 1982, cap. 6.4

37. Resolução do Conselho Nacional do Meio Ambiente, $N^{\circ} 357,17 / 03 / 2005$; Diário Oficial da União, 18/03/2005.

38. Andersson, S.; Collén, B.; Kruuse, G.; Kuylenstierna, U.; Acta Chem. Scand. 1957, 11, 1653.

39. Kumari, J. E.; Berckman, S.; Yegnaraman, V.; Mohandas, P. N.; Hydrometallurgy 2002, 65, 217.

40. Baes Jr., C. F.; Zingaro, R. A.; Coleman, C. F.; J. Phys. Chem. 1958, 62, 129. 


\section{EXTRAÇÃo LÍQUIDO-LÍQUIDO DE FERRO(III) E TITÂNIO(IV) PELO ÁCIDO BIS-(2-ETIL-HEXIL) FOSFÓ- RICO (D2EHPA) EM MEIO DE ÁCIDO SULFÚRICO}

\section{Glauco Corrêa da Silva e José Waldemar Silva Dias da Cunha}

Departamento de Química e Materiais Nucleares, Instituto de Engenharia Nuclear, CP 68456, 21941-909 Rio de Janeiro - RJ, Brasil

Jo Dweck

Departamento de Processos Inorgânicos, Escola de Química, Universidade Federal do Rio de Janeiro, Av. Athos da Silveira Ramos, 149, CT, Bloco E, 21941-909 Rio de Janeiro - RJ, Brasil

Julio Carlos Afonso*

Departamento de Química Analítica, Instituto de Química, Universidade Federal do Rio de Janeiro, Av. Athos da Silveira Ramos, 149, CT, Bloco A, 21941-909 Rio de Janeiro - RJ, Brasil

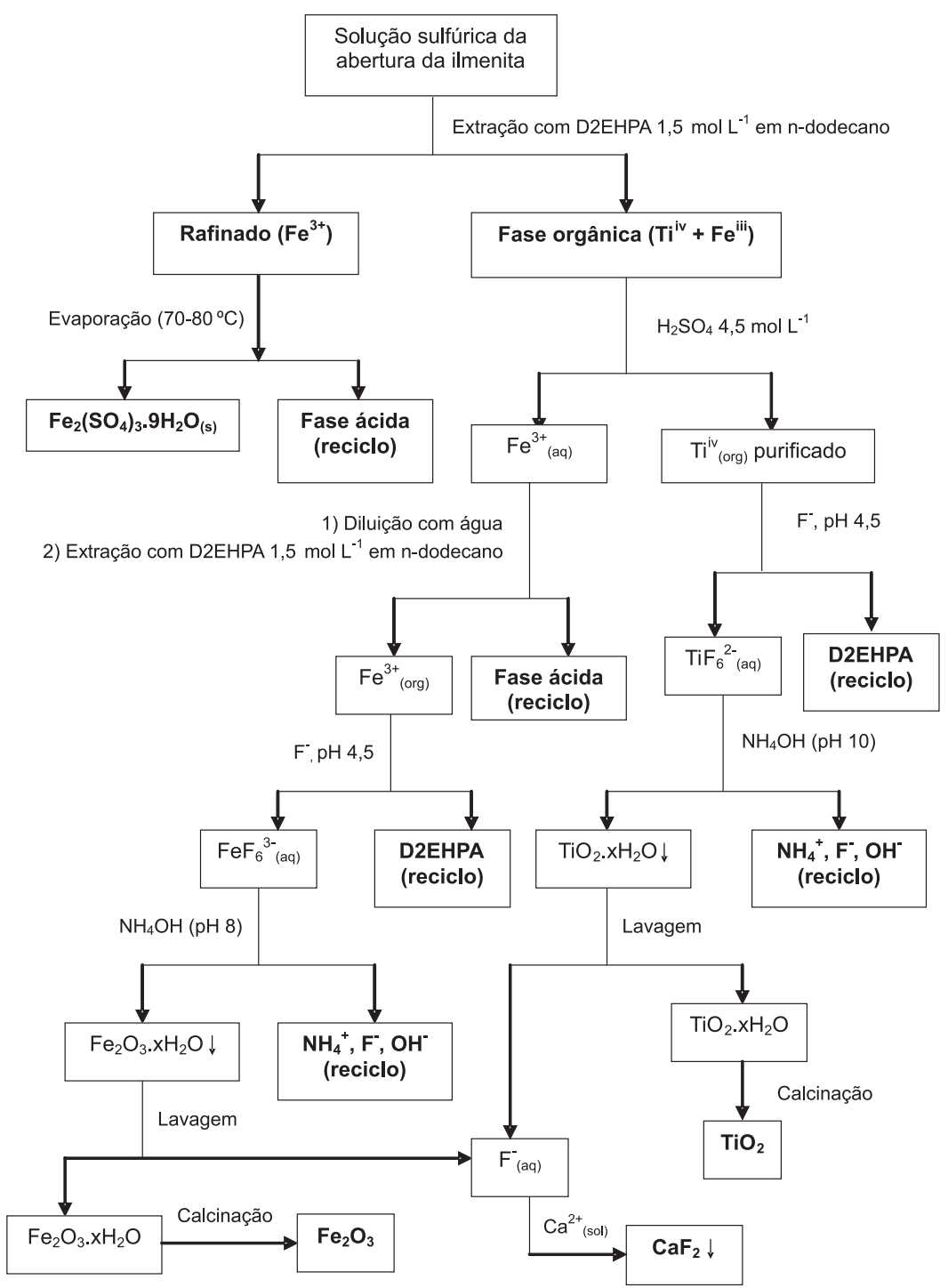

Figura 1S. Fluxograma ilustrativo da separação Fe-Ti de ilmenita tratada com $\mathrm{H}_{2} \mathrm{SO}_{4}$ 